\title{
ESTUDO DA EVOLUÇÃO DO COEFICIENTE GLOBAL DE TRANSFERÊNCIA DE CALOR EM FUNÇÃO DA COMPOSIÇÃO QUÍMICA DE LIGAS AI-Sn*
}

\author{
Ádria Rocha Corrêa ${ }^{1}$ \\ Kléber Agustin Sabat da Cruz²
}

\section{Resumo}

Durante a solidificação de ligas metálicas, o produto fundido se contrai, o qual provoca o surgimento de espaçamentos vazios na interface metal/molde chamados de "gaps". Estes gaps causam o aumento da resistência térmica influenciando em toda a cinética da solidificação. A transferência de calor durante a solidificação depende do conhecido parâmetro chamado de coeficiente global de transferência de calor $\left(\mathrm{h}_{\mathrm{g}}\right)$. Este parâmetro é representado pela equação do tipo: $h_{g}=C \cdot t^{-n}$, a qual indica que a eficiência térmica na interface metal/molde decai com o tempo. Muitos parâmetros podem influenciar o coeficiente global de transferência de calor $\left(h_{g}\right)$, neste estudo, os percentuais de Sn em ligas do sistema Al-Sn são analisados (10,20,30 e 40\%Sn). Portanto, o principal objetivo deste estudo é propor uma relação matemática que descreva, para o sistema Al-Sn, a evolução $\mathrm{h}_{\mathrm{g}}$ em função do teor de $\mathrm{Sn}$. A relação matemática foi obtida através do método dos mínimos quadrados

Palavras-chave: Solidificação; Al-Sn; Coeficiente global de transferência de calor.

\section{THEORICAL RESEARCH ON THE EVOLUTION OF THE GLOBAL HEAT TRANSFER COEFFICENT AS A FUNCTION OF THE Sn CONTENT IN AI-Sn ALLOYS}

\begin{abstract}
During the solidification of metallic alloys, the casting shrinks, which generates the rising of gaps at the metal/mold interface. These gaps cause the thermal resistance to become higher, influencing in all solidification kinetics. The heat transfer during solidification depends on the so-called global heat transfer coefficient $\left(\mathrm{h}_{\mathrm{g}}\right)$. This parameter is represented by the power function: $h_{g}=C . t^{-n}$, which indicates that the heat transfer efficiency falls down with the time. Many parameters can influence on the global heat transfer coefficient $\left(h_{g}\right)$, in this study, the Sn content in Al-Sn alloys $(10,20$, 30 and $40 \mathrm{wt} \% \mathrm{Sn}$ ) is analyzed. Therefore, this study has the main objective to find a mathematical relationship for Al-Sn alloy system that describes the evolution of the heat transfer coefficient $\left(h_{g}\right)$ as a function of the Sn content. The mathematical relationship was obtained by using the method of least squares that relates the coefficient $h_{g}$ and the Sn content.
\end{abstract}

Keywords: Solidification; Al-Sn; Global heat transfer coefficient.

1 Graduando do Programa de Ciência e Tecnologia, IEG-UFOPA. Bolsista do Programa Institucional de Bolsas de Iniciação Científica - PIBIC/FAPESPA. Santarém, Pará, Brasil.

2 Prof. Dr. Kléber Agustin Sabat da Cruz, IEG-UFOPA. Santarém, Pará, Brasil. 


\section{INTRODUÇÃO}

\subsection{O Sistema Al-Sn:}

O sistema binário Al-Sn é caracterizado por uma miscibilidade extremamente baixa do $\mathrm{Sn}$ no $\mathrm{Al}$, e que se situa abaixo de $0,09 \%$ em peso como pode ser visto na Figura 1-b. Nestas condições, as ligas com concentrações de Sn maiores do que 0,09\% são constituídas de uma estrutura metalográfica heterogênea caracterizada por uma matriz de alumínio com partículas de estanho disseminadas ao longo da matriz. Este tipo de arranjo estrutural determina o comportamento tribológico da liga, com a matriz tenaz sendo responsável pela resistência mecânica, enquanto as partículas de Sn atuam como um lubrificante sólido [7]. Esta classe de ligas apresenta um longo histórico de aplicações na fabricação de mancais, em função da combinação de resistência mecânica, boa conformabilidade e boas características de superfície. A liga mais comumente usada para este tipo de aplicação é a Al $20 \% \mathrm{Sn}$, em peso, que reúne este conjunto de características mencionadas embora tenha um custo relativamente alto [5].Embora as ligas Al-Sn aplicadas em mancais exibam as características acima mencionadas, com o desenvolvimento de motores cada vez mais velozes e submetidos e esforços crescentes e sobrecargas, o nível de resistência destas ligas não tem sido suficiente para atender estas novas necessidades de resistência à fadiga e ao desgaste. Uma das tendências nos estudos atuais consiste na adição de terceiros elementos na busca de um melhor compromisso entre estas propriedades, como é o caso da adição de Si [12]. Também para esta classe de ligas inexistem na literatura trabalhos que correlacionem parâmetros de microestrutura dendrítica (Figura 1-a) com propriedades mecânicas e resistência ao desgaste.

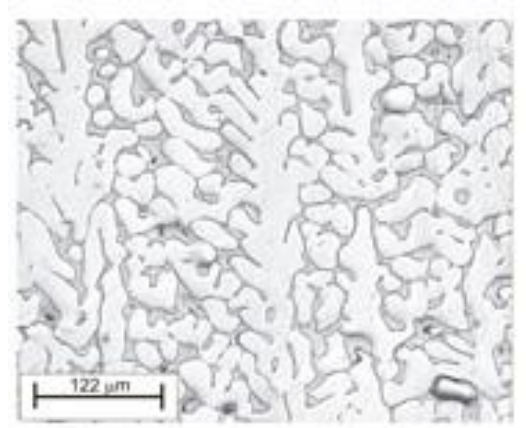

(a)

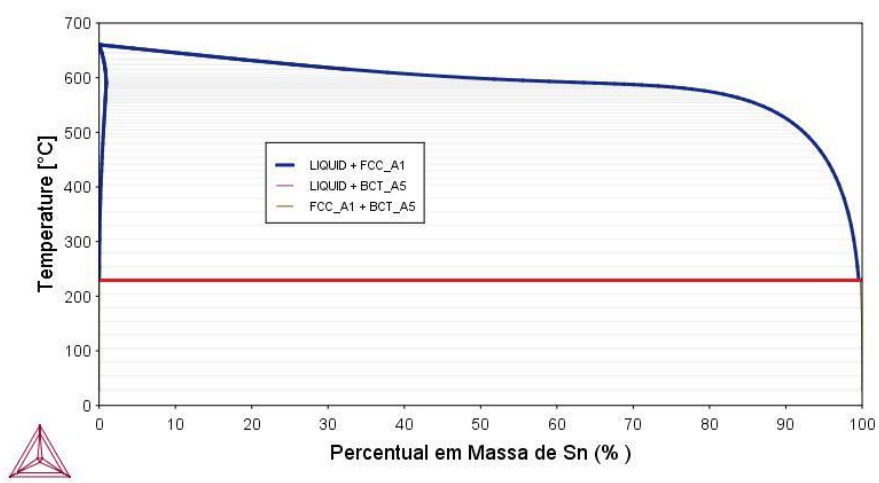

(b)

Figura 1. A- Microestrutura dendrítica de uma liga Al-40\%Sn solidificada unidirecionalmente [4,5]; B - Diagrama de fases Al-Sn [11]

\subsection{O Coeficiente Global de Transferência de Calor $\left(h_{g}\right)$ :}

Os resultados abordados neste estudo foram obtidos de um sistema de solidificação unidirecional projetado de tal modo que a extração de calor é realizada somente pela parte inferior refrigerada a água, promovendo solidificação direcional vertical [2]. 0 uso desta configuração experimental permite minimizar a convecção natural, bem como a convecção de soluto devido a correntes convectivas se o soluto rejeitado induzir uma massa específica do líquido interdendrítico maior do que a do volume global da liga fundida. 
A parte inferior do molde em contato com o metal, apresenta uma interface caracterizada por um coeficiente de transferência de calor metal/molde. Este coeficiente de transferência de calor representa um coeficiente de transferência de calor global que compreende todas as resistências térmicas entre o metal em solidificação e a água de refrigeração. A existência deste coeficiente de transferência de calor demanda a formulação de uma nova condição de contorno além da limitada pela fase pastosa.

O comportamento do coeficiente de transferência de calor global é traduzido como uma curva em decaimento exponencial ao longo do tempo (t) [3], comportamento este influenciado pela evolução da formação do gap mostrado na Figura 2.

Linhas de Fluxo de Calor

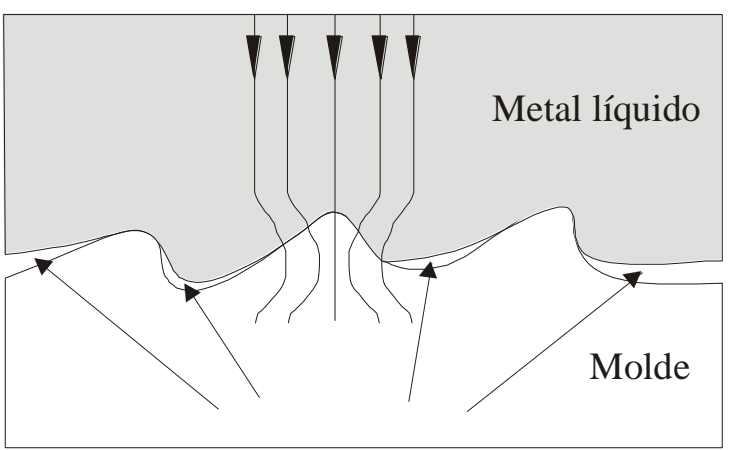

Estágio 1

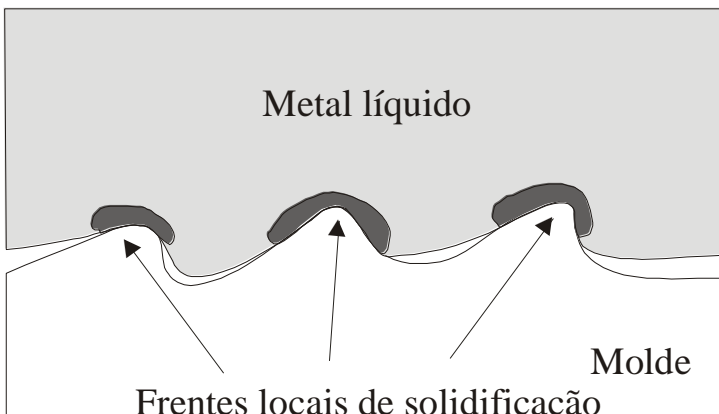

Estágio 3

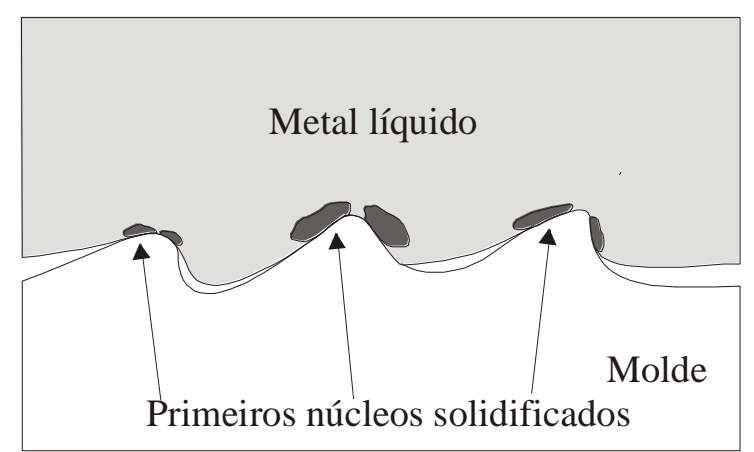

Estágio 2

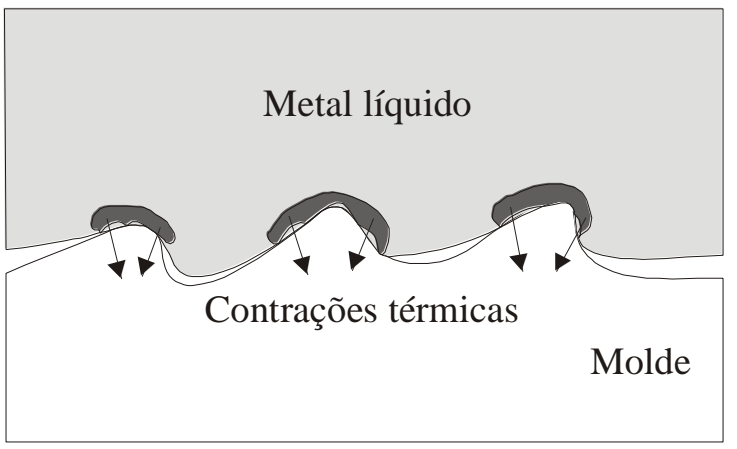

Estágio 4

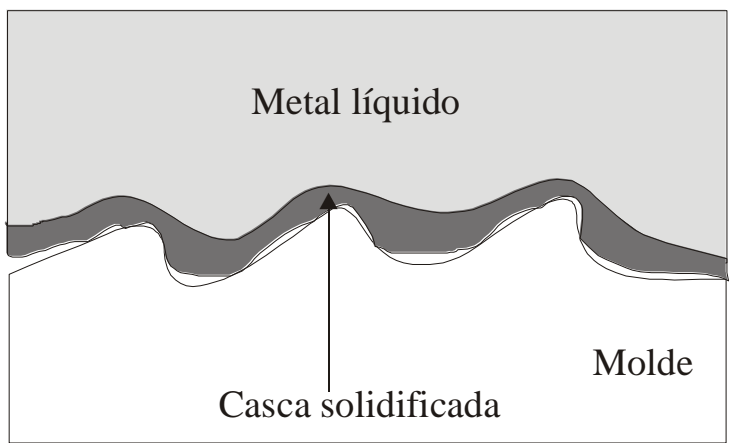

Estágio 5

Figura 2. Processo de Formação do "gap" na interface metal/molde [4]. 


\section{MÉTODOLOGIA}

Os resultados deste estudo foram baseados nos experimentos obtidos para ligas do sistema AI-Sn [1,2]. O modelo matemático se baseia no método de diferenças finitas que simula as condições de solidificação unidirecional. Este modelo foi introduzido inicialmente por Spim e Garcia [9] e utilizado posteriormente por Siqueira et al. [8]. Neste modelo, faz-se uma estimativa das constantes (multiplicador e expoente) que formam a equação (tipo potência- $h_{g}=C . t^{-n}$ ) transitória do coeficiente $h_{g}$, a qual resulta em um perfil térmico calculado que é confrontado com o perfil experimental, no caso de concordância chega-se ao resultado que representa o coeficiente que atuou durante a solidificação da liga, de acordo com a Figura 3.

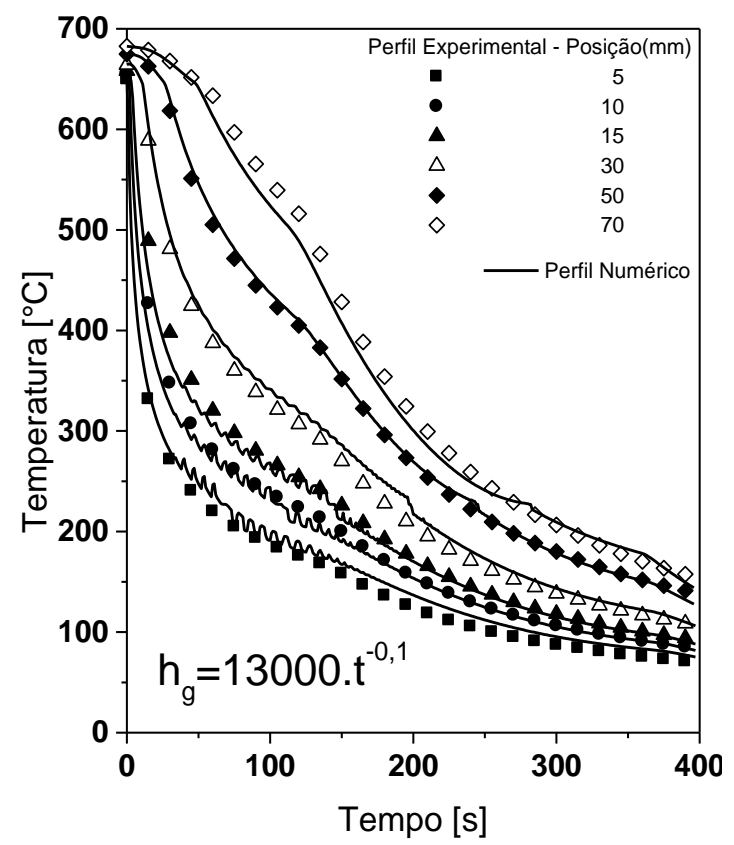

Figura 3. Confronto entre o perfil térmico experimental e calculado pelo método de diferenças finitas.

\section{RESULTADOS}

$\mathrm{Na}$ Figura 4, apresentam-se os resultados experimentais utilizados neste estudo [1,2]. O coeficiente $h_{g}$, tem uma tendência de reduzir seu valor até o percentual de $30 \% \mathrm{Sn}$ e posteriormente aumentar. Provavelmente este comportamento deve estar relacionado à imiscibilidade entre o $\mathrm{Al}$ e o $\mathrm{Sn}$, o que é característico das ligas do sistema Al-Sn. Comportamento diferente foi encontrado em ligas hipoeutéticas do sistema Al-Si, onde a medida que o percentual de Si se aproximou da concentração eutética, houve a redução do multiplicador do coeficiente $h_{g}[10]$. 


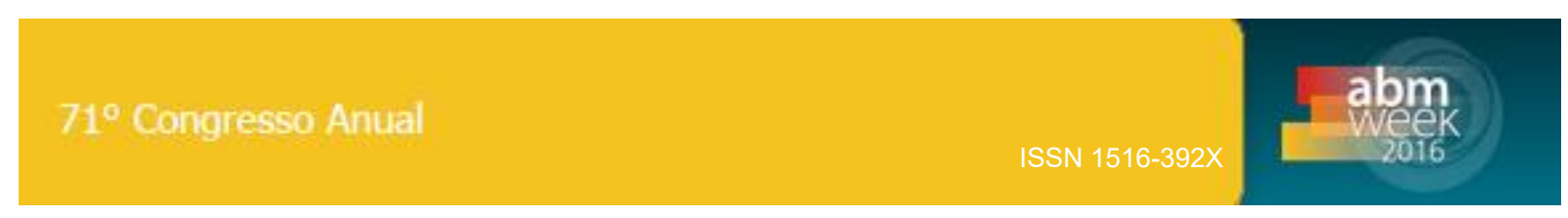

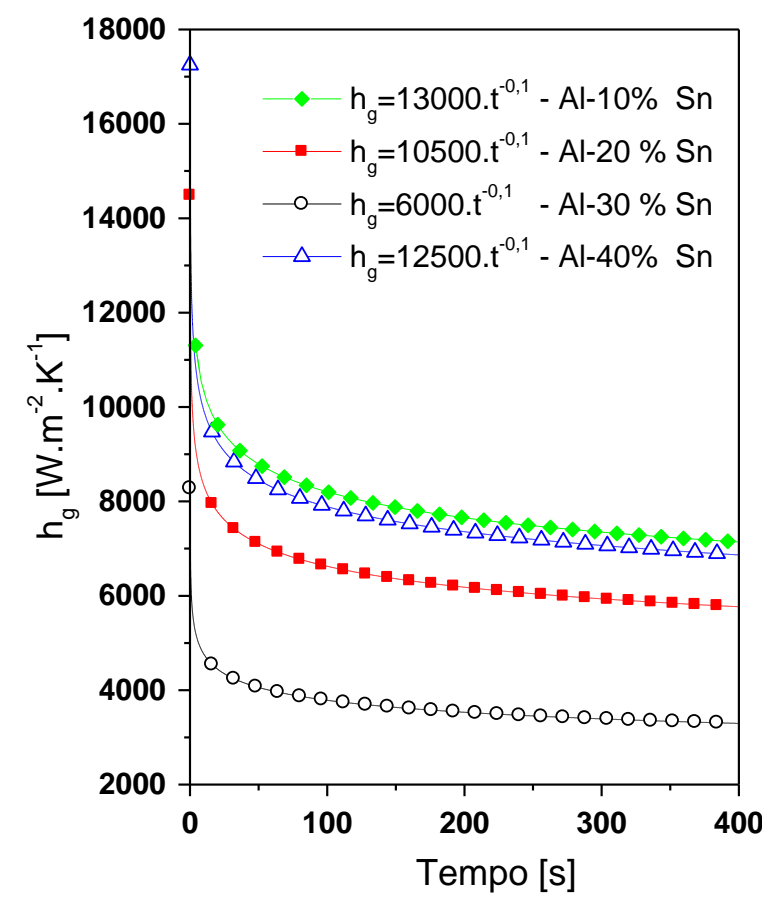

(A)

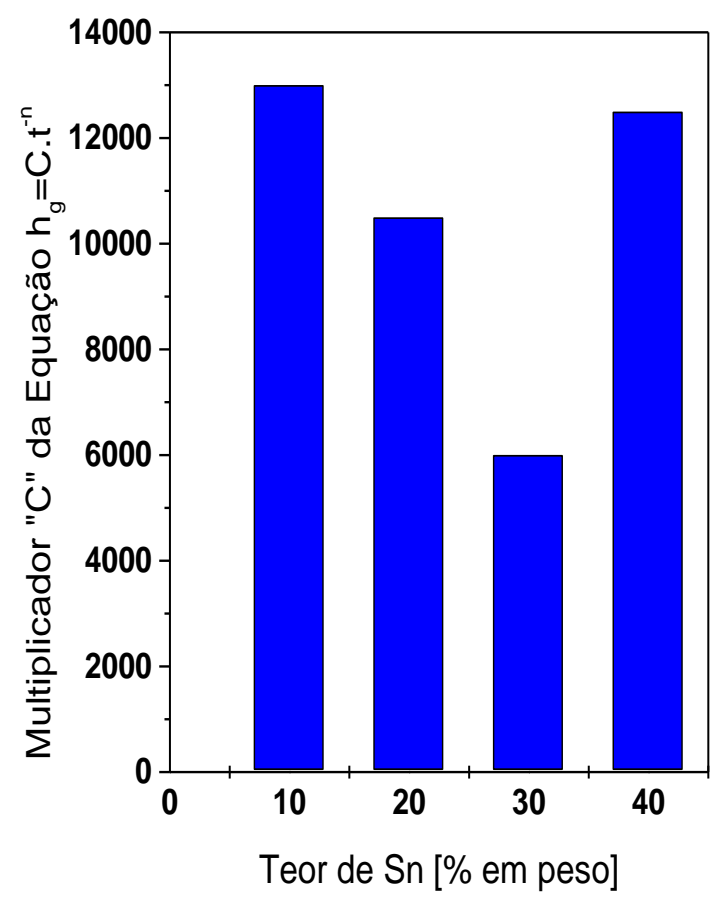

(B)

Figura 4. (A) Coeficientes transitórios de transferência de calor para ligas Al-Sn solidificadas na forma vertical ascendente em molde de aço carbono 1020 refrigerado a água, com espessura de 3 $\mathrm{mm}$ e superaquecimento de $2^{\circ} \mathrm{C}$; (B) Gráfico mostrando a evolução do multiplicador "C" com o aumento do teor de Sn [1,2].

Inicialmente, calculou-se os parâmetros de qualidade do ajuste de mínimos quadrados à função $h_{g}=f(\% C)$. os dados foram também submetidos a escalas logarítmicas e semilogarítmicas de y para verificação da qualidade dos ajustes exponencial e potência $\left(y=b \cdot x^{m} ; y=b \cdot 10^{m x}\right)$. Notou-se que a linearidade dos dados não existiu, fato este que indica que estas funções não representam a função $h_{g}=f(\% S n)$. Os parâmetros de qualidade de ajuste são apresentados na tabela 1.

Tabela 1. Parâmetro de qualidade de ajuste por mínimos quadrados

\begin{tabular}{|l|l|l|l|}
\hline $\begin{array}{c}\text { Grau do polinômio } \\
\text { de ajuste }\end{array}$ & $\begin{array}{c}\text { Soma do quadrado } \\
\text { dos desvios }(\mathrm{S})\end{array}$ & $\begin{array}{c}\text { Soma do quadrado } \\
\text { dos resíduos }(\mathrm{J})\end{array}$ & $\begin{array}{c}\text { Coeficiente de } \\
\text { determinação } \mathrm{r}^{2}\end{array}$ \\
\hline 1 & $3,05 \times 10^{7}$ & $2,87 \times 10^{7}$ & 0,059 \\
\hline 2 & $3,05 \times 10^{7}$ & $8,45 \times 10^{7}$ & 0,723 \\
\hline 3 & $3,05 \times 10^{7}$ & $9,06 \times 10^{-22}$ & 0,998 \\
\hline
\end{tabular}

$\mathrm{Na}$ Figura 5 tem-se o ajuste polinomial cúbico para a relação entre o coeficiente $\mathrm{h}_{\mathrm{g}} \mathrm{e}$ o teor de Sn. Com base na Tabela 01, os melhores parâmetros foram para o ajuste cúbico, portanto pode-se propor que na faixa de composição entre 10 a $40 \%$ Sn a função que melhor descreve a evolução do coeficiente $h_{g}$ em função do teor de Sn é a equação 01.

$$
y=2,2 \cdot x^{3}-1,4 \cdot 10^{2} \cdot x^{2}+2,4 \cdot 10^{3} \cdot x+5 \cdot 10^{2}
$$



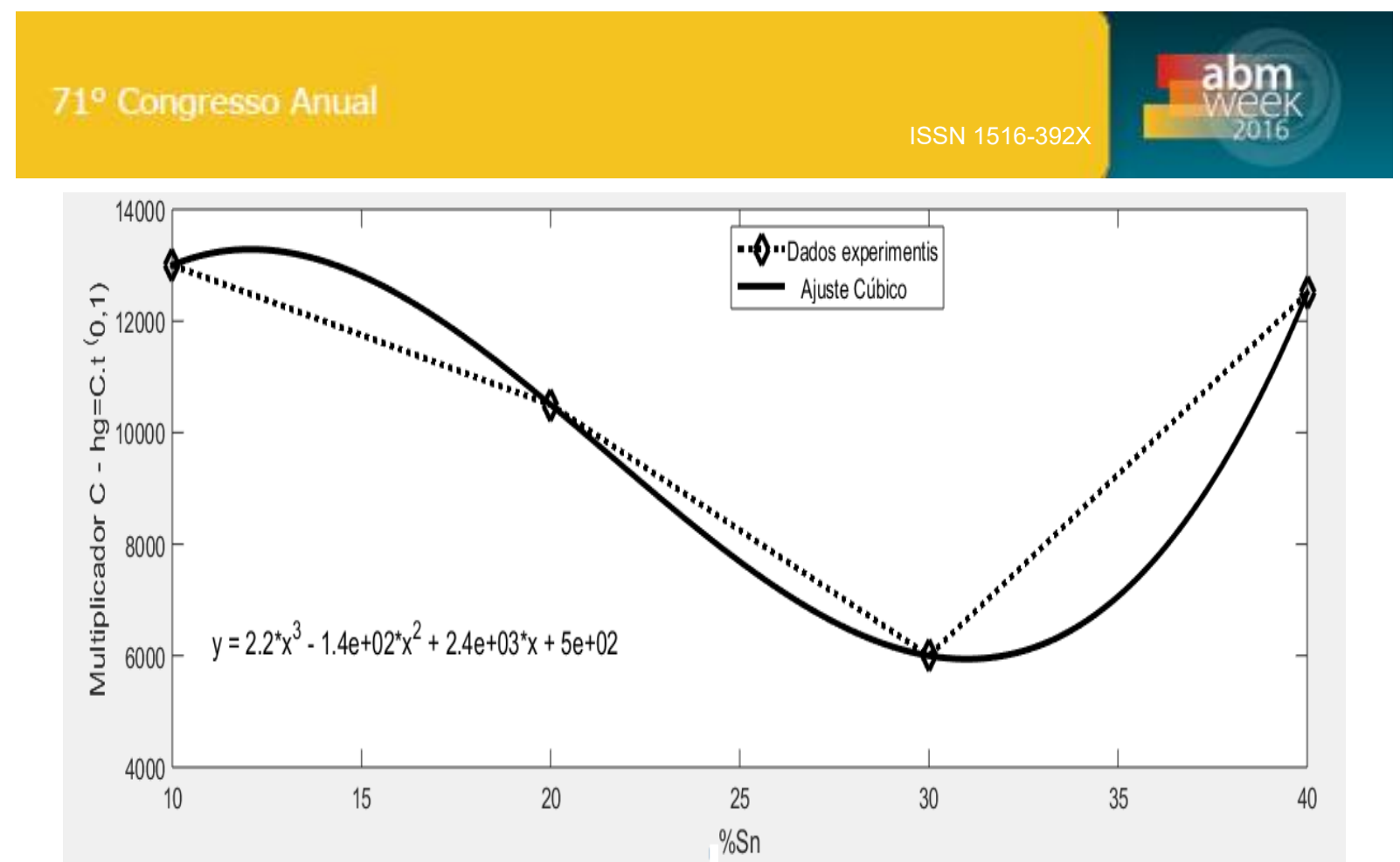

Figura 5. Curva do ajuste cúbico para a função $h_{g}=f(\% S n)$.

\section{CONCLUSÃO}

A função que melhor se adequou para representar a evolução do coeficiente $h_{g}$ em função do teor de Sn, foi:

$$
y=2,2 \cdot x^{3}-1,4 \cdot 10^{2} \cdot x^{2}+2,4 \cdot 10^{3} \cdot x+5 \cdot 10^{2}
$$

O método usado para o ajuste de curva foi o método dos mínimos quadrados, após a verificação em escalas semilogarítmica de y e logarítmica que as funções potência e exponencial não descrevem a relação $h_{g}=f(\% S n)$, devido a falta de linearidade dos dados.

\section{Agradecimentos}

Agradeço a FAPESPA pelo apoio financeiro dado para o desenvolvimento deste trabalho no ano de 2015.

\section{REFERÊNCIAS}

1 CRUZ, Kleber Agustin Sabat da,. Microestrutura de solidificação, resistência mecânica e ao desgaste de ligas Al-Sn e Al-Si - Tese de doutorado - UNICAMP/FEM -

Campinas - SP,2008.

2 CRUZ, K.S., et al. Dendritic arm spacing affecting mechanical properties and wear behavior Al-Sn and Al-Si alloys directionally solidified under unsteady-state conditions ,Metallurgical and Materials Transactions, v.41A, p. 972-984, 2010.

3 KRISHNAN, M. \& SHARMA, D. G. R. Determination of the interfacial heat transfer coefficient $h$ inunidirectional heat flow by Beck's nonlinear estimation. procedure International Communications in Heat Mass Transfer", v. 23, n. 2, pp. 203214, 1996.

4 LOLOU, T.; Artyukhin, E.A.; BARDON, J. P. Estimation of therma, contact resistance during the first stages of metal solification process: II- experiment principle and modelisation, International Journal of Heat Mass Transfer, v.42, 1999b, pp.2129-2142. 
5 PATHAK, J. P.; MOHAN, S. Tribological Behavior of Conventional Al-Sn and Equivalent Al-Pb Alloys Under Lubrication, Bulletin on Materials Science, vol. 26, pp. 315-320, 2003.

6 PERES, M. D.; SIQUEIRA, C. A.; GARCIA, A. Macrostructural and microstructural development in A-Si alloys directionally solidified under unsteady-state conditions. Journal of Alloys and Compounds, v. 381, pp. 168-181, 2004.

7 PERRONE, A. et al. Al-Sn Thin films Deposited by Pulsed Laser Ablation, Materials Science and Engineering C, vol. 22, pp. 465-468, 2002.

8 QUARESMA, J. M. V.; SANTOS, C.A.; SPIM Jr., J.A. \& GARCIA, A. Influência do Material e da espessura da Parede do Molde nos Coeficientes de Transferência de Calor nas Interfaces Metal/Molde e Molde/Ambiente, CD do II Congresso Internacional de Tecnologia Metalúrgica e de Materiais - ABM 97, São Paulo- SP, 1997.

9 SANTOS, C. A.; QUARESMA J. M. V.; GARCIA A.; Determination of transient interfacial heat transfer coefficients in chill mold casting, Journal of Alloys and Compounds, v. 319, pp. 174-186, 2001.

10 SPIM, J. A.; GARCIA A.; A modified network approach for modeling solidification of complex-shaped domains, Numerical Heat Transfer- B, v. 38, pp. 75-92, 2000.

11 Thermo-calc software, v.4, Stockholm, Sweden, 2008.

12 YUAN, G.C.; LI, Z.J.; LOU, Y.X.; ZHANG, X.M. Study on Crystallization and Microstructure for New Series of Al-Sn-Si Alloys, Materials Science and Engineering A, vol. 280, pp. 108-115, 2000. 